\title{
Inverted harmonic oscillator dynamics of the nonequilibrium phase transition in the Dicke model
}

\author{
Karol Gietka $\odot^{*}$ and Thomas Busch $\odot$ \\ Quantum Systems Unit, Okinawa Institute of Science and Technology Graduate University, Onna, Okinawa 904-0495, Japan
}

(Received 30 June 2021; accepted 14 September 2021; published 29 September 2021)

\begin{abstract}
We show how the dynamics of the Dicke model after a quench from the ground-state configuration of the normal phase into the superradiant phase can be described for a limited time by a simple inverted harmonic oscillator model and that this limited time approaches infinity in the thermodynamic limit. Although we specifically discuss the Dicke model, the presented mechanism can also be used to describe dynamical quantum phase transitions in other systems and presents an opportunity for simulations of physical phenomena associated with an inverted harmonic oscillator.
\end{abstract}

DOI: 10.1103/PhysRevE.104.034132

\section{INTRODUCTION}

Quantum phase transitions describe abrupt changes of a system's properties while varying physical parameters, such as magnetization, at absolute zero temperature [1]. Depending on how fast the parameter is being changed, one can distinguish equilibrium and nonequilibrium quantum phase transitions and despite an almost dialectical relationship between these two types [2], the nonequilibrium one remains less well understood compared to the equilibrium one [3-6]. The typical framework for studying nonequilibrium quantum phase transitions is a sudden change of some physical parameter in the Hamiltonian [7-13]. After such a quench, a system that was initially prepared in the ground state becomes a superposition of eigenstates of the quenched Hamiltonian which subsequently drives the evolution. In a complex system, such as the Dicke model, which consists of a collective spin coupled to a harmonic oscillator [14], one could expect a quench to lead to a complicated dynamical behavior, in certain cases, behavior with signatures of quantum chaos [15-18].

In this work we show that for a limited and quantifiable time the dynamics after a quench can be mapped to the dynamics of a simple inverted harmonic oscillator $[19,20]$. Our model is based on an effective Hamiltonian that cannot be used to fully describe the equilibrium states [21,22]. However, we show that it correctly describes the dynamics for a limited time and under certain conditions. In fact, we show that in the thermodynamic limit the nonequilibrium Dicke phase transition becomes equivalent to a harmonic oscillator with a tunable frequency on both sides of the phase transition. By manipulating the parameters of the system and the spin polarization of the initial state, this tunable frequency can

\footnotetext{
*Corresponding author: karol.gietka@oist.jp
}

Published by the American Physical Society under the terms of the Creative Commons Attribution 4.0 International license. Further distribution of this work must maintain attribution to the author(s) and the published article's title, journal citation, and DOI. change from being purely real (normal phase), through being zero (critical point), to being purely imaginary (superradiant phase). Using state-of-the-art tools from quantum simulation $[23,24]$, we give a precise description of how to observe the physics of the inverted oscillator even far away from the thermodynamic limit. Moreover, our proposal provides an opportunity for simulations of physical phenomena associated with an inverted harmonic oscillator [20].

\section{DICKE MODEL}

The Dicke model is a paradigmatic model describing the interaction of $N$ spin- $\frac{1}{2}$ systems (in general, two-level systems) with an energy splitting of $\Omega$ with a single-mode field of frequency $\omega[14,25-28]$. Its Hamiltonian can be written as (we set $\hbar=1$ throughout the paper)

$$
\hat{H}=\omega \hat{a}^{\dagger} \hat{a}+\Omega \hat{S}_{z}+\frac{g}{\sqrt{N}}\left(\hat{a}+\hat{a}^{\dagger}\right) \hat{S}_{x},
$$

where we have used the collective spin operators $\hat{S}_{i}=$ $\sum_{n=1}^{N} \hat{\sigma}_{i}^{(n)} / 2$, with $\hat{\sigma}_{i}^{(n)}$ the $i$ th Pauli matrix of the $n$th spin, and bosonic field creation and annihilation operators $\hat{a}^{\dagger}$ and $\hat{a}$, respectively, satisfying the bosonic commutation relations $\left[\hat{a}, \hat{a}^{\dagger}\right]=\hat{\mathbb{1}}$. The parameter $g$ quantifies the collective interaction of $N$ spins with the single-mode field. At a critical coupling strength $g=g_{c} \equiv \sqrt{\omega \Omega}$ in the limit of $\sqrt{\Omega N / \omega} \rightarrow$ $\infty$ the Dicke model exhibits a quantum phase transition into a superradiant state. For $N \rightarrow \infty$ this limit becomes thermodynamic [14], which means that the quantum phase transition occurs for an arbitrary but finite ratio of $\Omega / \omega$. To gain more insight into the dynamics one can apply the unitary transformation $\hat{U}=\exp \left[i(g / \sqrt{N} \Omega)\left(\hat{a}+\hat{a}^{\dagger}\right) \hat{S}_{y}\right]$ to the Hamiltonian in Eq. (1) and obtain an effective description [22,29]

$$
\hat{H}_{\mathrm{eff}} \simeq \omega \hat{a}^{\dagger} \hat{a}+\Omega \hat{S}_{z}+\frac{g^{2}}{2 \Omega N}\left(\hat{a}+\hat{a}^{\dagger}\right)^{2} \hat{S}_{z},
$$

which is exact in the limit of $\sqrt{\Omega N / \omega} \rightarrow \infty$. However, it is generally believed that this effective Hamiltonian is only applicable in the normal phase when $g<g_{c}$ [22]. As the energy gap is given by $\omega \sqrt{1-g^{2} / g_{c}^{2}}$, the ground-state energy 
(a)

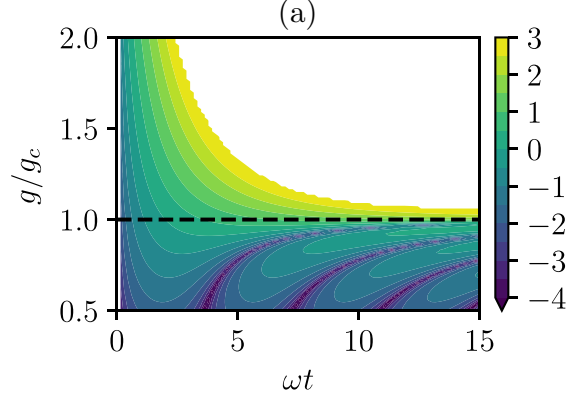

(b)

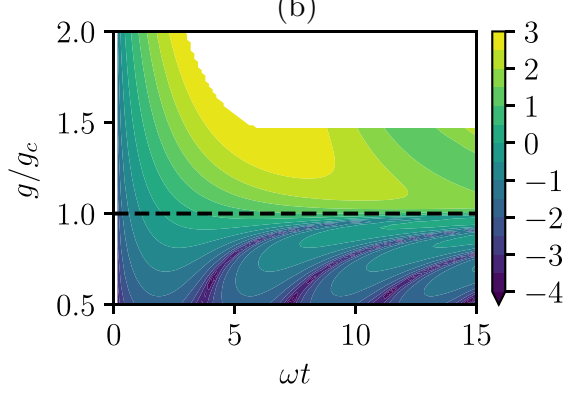

(c)

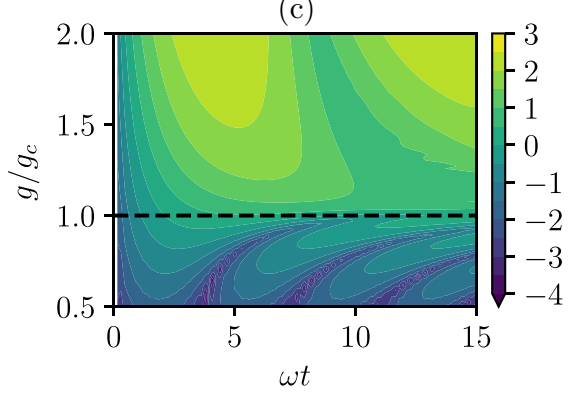

FIG. 1. Logarithm of the average number of photons as a function of $g / g_{c}$ and $t$ for (a) $\sqrt{\Omega N / \omega}=100$, (b) $\sqrt{\Omega N / \omega} \approx 31.6$, and (c) $\sqrt{\Omega N / \omega}=10$. The black dashed line corresponds to the critical coupling in the thermodynamic limit. White regions correspond to unreliable numerical simulations after the boundary of the Hilbert space (set to $n=3000$ ) has been reached. For simplicity, in the numerical simulations we set $N=1$.

of the effective Hamiltonian is imaginary for $g>g_{c}$ and a different effective description has to be employed in the superradiant phase. However, in this work we argue that, following a quench from the ground state in the normal phase to the superradiant phase in the thermodynamic limit, the effective Hamiltonian from Eq. (2) can also be the correct description in the superradiant phase as in the latter the ground-state energy tends to $-\infty$ (similarly to the Holstein-Primakoff representation of the Dicke model [30]). Moreover, even away from the thermodynamic limit $(\sqrt{\Omega N / \omega} \gg 1)$, one can use the Hamiltonian (2) to describe the dynamics following a sudden quench to $g>g_{c}$ for times shorter than some critical time related to the initial state and energy of the ground state in the superradiant phase.

The initial state (ground state for $g=0$ ) consists of the vacuum state of the field $|0\rangle(\hat{a}|0\rangle=0)$ and the collective spin-down state, which is the eigenstate of the $\hat{S}_{z}$ operator with minimal eigenvalue, i.e., $\hat{S}_{z}|\psi\rangle=-\frac{N}{2}|\psi\rangle$. It can be easily checked that the transformation $\hat{U}=\exp [i(g / \sqrt{N} \Omega)(\hat{a}+$ $\left.\left.\hat{a}^{\dagger}\right) \hat{S}_{y}\right]$ leaves that state unchanged once $(\omega / \Omega)\left(g^{2} / g_{c}^{2}\right) \ll 1$ (see Appendix A for a derivation). Therefore, since the effective Hamiltonian conserves the projection of the spin onto the $z$ axis, we can replace the operator with its lowest eigenvalue leading to

$$
\hat{H}=\left(\omega-\frac{g^{2}}{2 \Omega}\right) \hat{a}^{\dagger} \hat{a}-\frac{g^{2}}{4 \Omega}\left(\hat{a}^{2}+\hat{a}^{\dagger 2}\right) .
$$

The above Hamiltonian describes a single-mode field with a modified frequency $\omega-g^{2} / 2 \Omega$ which is being squeezed with a strength equal to $g^{2} / 2 \Omega$. Both quantities are independent of $N$ and the two noncommuting mechanisms give rise to rich physics. We now consider a sudden quench from $g=0$ to some nonzero $g$. For $g$ below the critical value, the vacuum state is being squeezed and the number of photons increases [31]. However, the additional rotation mechanism causes the state to unsqueeze after a $\pi / 2$ rotation and returns it to the vacuum with no photons (see Appendix C). When $g$ approaches the critical value, the squeezing mechanism becomes dominant exactly at the critical point $g=g_{c}$ [note that at the critical point the first term in Hamiltonian (3) does not vanish] and the initial vacuum state starts to get squeezed more and more with further increasing $g$. This can be seen in Fig. 1, where we plot the average number of photons as a function of $g / g_{c}$ and $t$ for various ratios of $\sqrt{\Omega N / \omega}$ and for an initial vacuum state evolved with the Hamiltonian from Eq. (1). Looking at Fig. 1, we can identify parameter regions of squeezing rotation (oscillations of the number of photons) below the critical point and for the case of $\sqrt{\Omega N / \omega}$ very large [see Fig. 1(a)] continuous squeezing (increase of the number of photons) above the critical point. Note that white regions in this plot correspond to unreliable numerical simulations after the boundary of the Hilbert space (set to $n=3000$ ) has been reached due to the large increase in photon production. However, going away from the limit $\sqrt{\Omega N / \omega} \rightarrow \infty$ [see Figs. 1(b) and 1(c)], the number of photons does not continuously increase in the superradiant regime, which puts a limit on the time for which the effective Hamiltonian from Eq. (2) can be used to describe the dynamics governed by Eq. (1) for $g>g_{c}$. The limiting time is heralded when the growth of the photon number slows down. However, for a limited time the Hamiltonian from Eq. (2) gives rise to the correct qualitative behavior even if $g>g_{c}$. Before discussing the time limitation for that description let us now have a look at the system from another perspective.

\section{INVERTED HARMONIC OSCILLATOR}

Additional insight can be gained by rewriting the Hamiltonian (3) in terms of the pseudoposition and pseudomomentum operators (quadratures) $\hat{X}=\left(\hat{a}+\hat{a}^{\dagger}\right) / \sqrt{2}$ and $\hat{P}=$ $\left(\hat{a}-\hat{a}^{\dagger}\right) / \sqrt{2} i$, respectively, as

$$
\hat{H}=\frac{\omega}{2} \hat{P}^{2}+\frac{1}{2 \omega}\left(\omega^{2}-\frac{\omega g^{2}}{\Omega}\right) \hat{X}^{2} .
$$

This shows that the system dynamics behaves as a particle with mass $1 / \omega$ in a harmonic oscillator potential of frequency $\omega \sqrt{1-g^{2} / g_{c}^{2}}$. If $g<g_{c}$ the frequency is real and the energy gap above the ground state is proportional to $\omega \sqrt{1-g^{2} / g_{c}^{2}}$. If $g>g_{c}$ the frequency becomes purely imaginary and the energy gap cannot be defined as there is no ground state of the Hamiltonian. In other words, for a quench from the $g=0$ ground state, the Dicke model in the thermodynamic limit becomes an inverted harmonic oscillator in the superradiant phase. With this picture in mind, we can easily explain the dynamics seen in the preceding section. As the coupling parameter is increased towards the critical value the frequency 
of the oscillations (squeezing rotation) decreases, which corresponds to the harmonic potential being flattened. At the critical point $g=g_{c}$ the frequency of the oscillations becomes zero as the harmonic potential becomes completely flat (free particle). As the coupling becomes greater than the critical coupling the frequency becomes purely imaginary or, in other words, the harmonic potential flips upside down, giving rise to an unbounded fall (squeezing). However, away from the thermodynamic limit, i.e., when $\sqrt{\Omega N / \omega} \gg 1$, this picture only applies for finite periods, and we will attempt to clarify the limitations in the following.

\section{CONNECTOR OPERATOR}

As discussed in the Introduction, we will use the tools from quantum simulation theory $[23,24]$ to get a deeper understanding of how applicable the inverted harmonic oscillator picture is. The condition for a quantum simulator can be expressed as

$$
\left\langle\psi\left|e^{i \hat{H}_{\mathrm{QS}} t} e^{-i \hat{H}_{\mathrm{T}} t}\right| \psi\right\rangle=\left\langle\psi\left|e^{i \hat{h}(t)}\right| \psi\right\rangle=e^{i \xi(t)},
$$

where $\hat{H}_{\mathrm{QS}}$ and $\hat{H}_{\mathrm{T}}$ are the simulator and target Hamiltonian, respectively, $\xi(t)$ is a real-valued function of time, and $\hat{h}(t)$ is the connector operator which can be expressed using the Baker-Campbell-Hausdorff formula as

$$
\hat{h}(t)=t\left(\hat{H}_{\mathrm{QS}}-\hat{H}_{\mathrm{T}}\right)+\frac{i t^{2}}{2}\left[\hat{H}_{\mathrm{QS}},-\hat{H}_{\mathrm{T}}\right]+\cdots,
$$

where $[\cdot, \cdot]$ stands for the commutator and the ellipsis indicates terms involving higher-order commutators of $\hat{H}_{\mathrm{QS}}$ and $\hat{H}_{\mathrm{T}}$. In general, for states $|\psi\rangle$ which are eigenstates of $h(t)$ but not eigenstates of $\hat{H}_{\mathrm{QS}}$ and $\hat{H}_{\mathrm{T}}$, the two Hamiltonians will realize the same dynamics. For the Dicke model (1) and the effective description from Eq. (2), the connector operator becomes exactly 0 in the thermodynamic limit. However, for a limited time the connector operator also converges to 0 , indicating the transitional validity of the Hamiltonian (2).

The condition for the time during which the Hamiltonian (2) is valid can be expressed as a relation between the instantaneous average number of photons and the number of photons in the ground state of the Dicke model. The latter can be calculated by rewriting the Hamiltonian using the pseudoposition and pseudomomentum operators

$$
\hat{H}=\frac{\omega}{2} \hat{p}^{2}+\frac{\omega}{2} \hat{x}^{2}+\Omega \hat{S}_{z}+\frac{\sqrt{2} g}{\sqrt{N}} \hat{x} \hat{S}_{x}
$$

The part containing the spin operators can be easily diagonalized and its ground-state energy can be found to be

$$
\left(\Omega \hat{S}_{z}+\frac{\sqrt{2} g}{\sqrt{N}} \hat{x} \hat{S}_{x}\right)|\sigma\rangle=\left(-\frac{1}{2} \sqrt{N} \sqrt{2 g^{2} \hat{x}^{2}+N \Omega^{2}}\right)|\sigma\rangle
$$

where $|\sigma\rangle$ is the spin ground state. Projecting the Hamiltonian onto the spin ground state yields

$$
\hat{H}=\frac{\omega}{2} \hat{p}^{2}+\frac{\omega}{2} \hat{x}^{2}-\frac{1}{2} \sqrt{N} \sqrt{2 g^{2} \hat{x}^{2}+N \Omega^{2}} .
$$

The above Hamiltonian describes a particle with mass $1 / \omega$ moving in a double-well potential given by $\hat{V}(x)=\frac{\omega}{2} \hat{x}^{2}-$ $\frac{1}{2} \sqrt{N} \sqrt{2 g^{2} \hat{x}^{2}+N \Omega^{2}}$. The minima of such an effective potential can be evaluated to be

$$
x_{0}= \pm \sqrt{\frac{N \Omega}{2 \omega}} \sqrt{\frac{g^{2}}{g_{c}^{2}}-\frac{g_{c}^{2}}{g^{2}}} .
$$

From a simple phenomenological argument (see Fig. 2) the critical position for the observation of the inverted harmonic oscillator will be a quarter of the minima position, i.e., $x_{c}=x_{0} / 4$.

According to this position, we can establish an energy relation which has to be satisfied

$$
\frac{\omega}{2}\left\langle\hat{p}^{2}+\hat{x}^{2}\right\rangle<\frac{\omega}{2} \hat{x}_{c}^{2}=\frac{\omega}{32} \hat{x}_{0}^{2}
$$

which upon simplification yields

$$
\left\langle\hat{a}^{\dagger} \hat{a}\right\rangle<\frac{1}{32} \frac{N \Omega}{\omega}\left(\frac{g^{2}}{g_{c}^{2}}-\frac{g_{c}^{2}}{g^{2}}\right) .
$$

For a special case of $g=\sqrt{2} g_{c}$ and initial state being the vacuum state, we can simplify the condition to $\sinh ^{2}(\omega t)<$ $\frac{3}{64}(N \Omega / \omega)$, which for $\omega t>1$ becomes

$$
t<t_{c} \equiv \ln \left[\sqrt{\frac{3}{16}} \sqrt{\frac{N \Omega}{\omega}}\right] \omega^{-1} .
$$

The above time limitations can be intuitively understood as a time until which the time-evolved state feels the bottom of the effective double-well potential after a quench out of the equilibrium position at $x=0$ (see Fig. 2). In the thermodynamic limit, the overlap increases as the minima of the double-well potential are located at $+\infty$ and $-\infty$, which
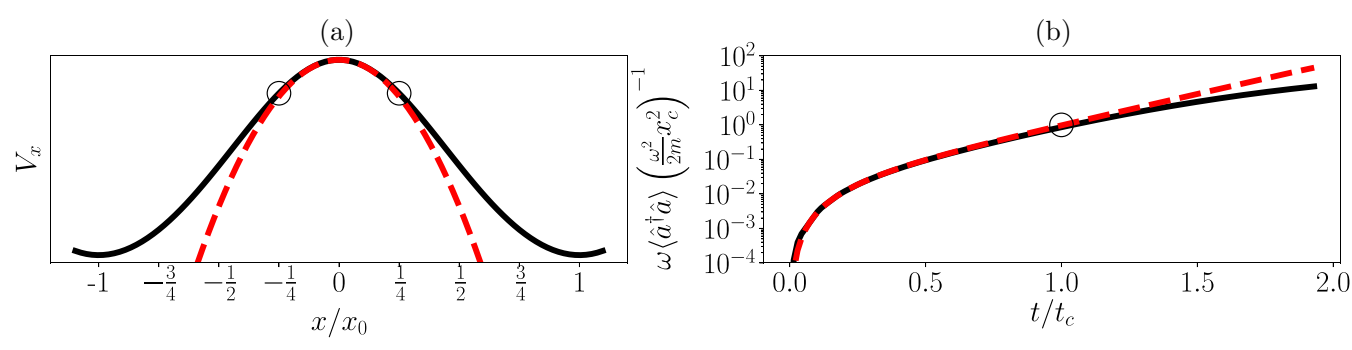

FIG. 2. Phenomenological condition for simulating the inverted oscillator with the Dicke model. (a) Effective double-well potential (black solid line) overlaid on an inverted oscillator potential (red dashed line). The black circles indicate the critical position until which both models agree. (b) The critical position can be translated to a condition on the number of photons which can be related to a critical time for which the Dicke model can be used as a simulator. This time is indicated by the black circle. 
(a)

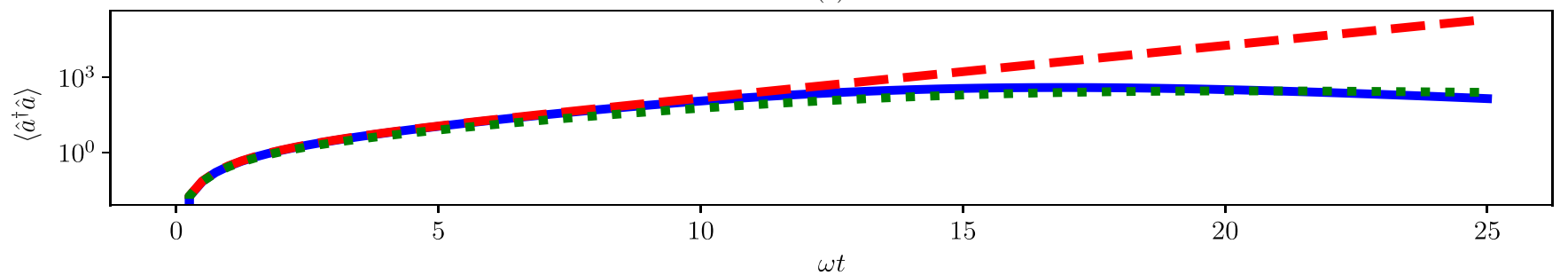

(b)

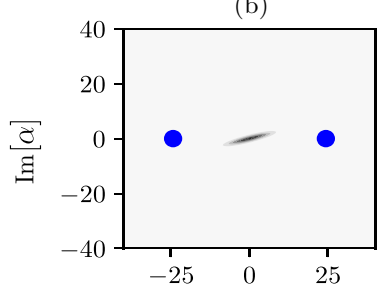

(g)

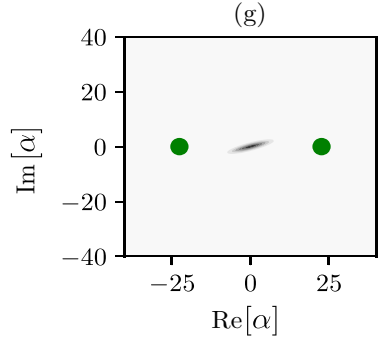

(c)

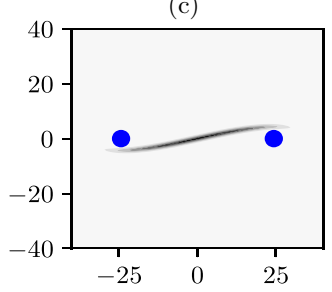

(h)

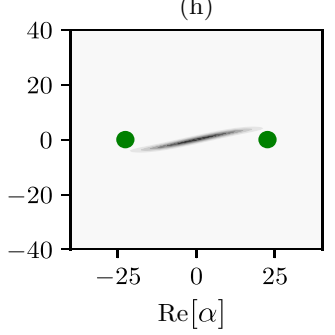

(d)

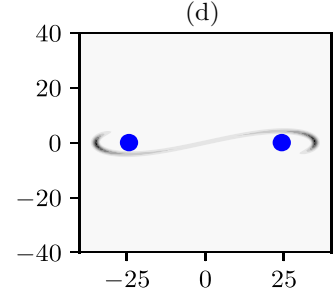

(i)

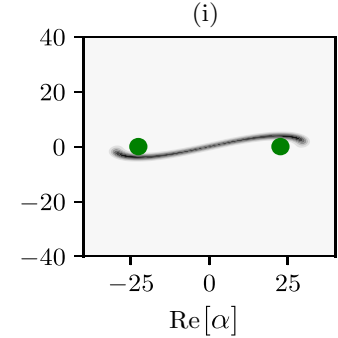

(e)

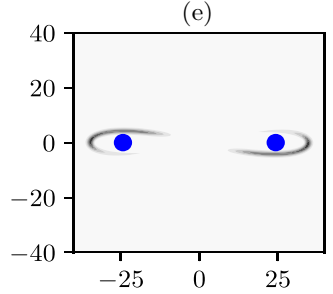

(j)

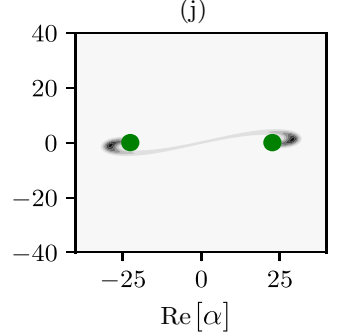

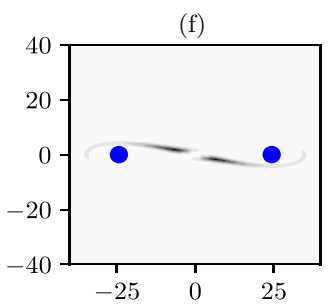

(k)

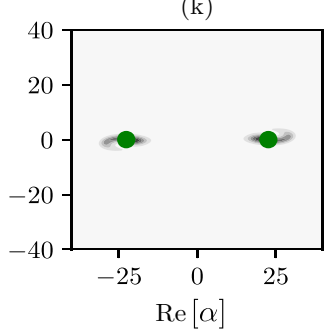

FIG. 3. (a) Comparison between an inverted harmonic oscillator (red dashed line) and the isolated (blue solid line) and open (green dotted line) Dicke models. The Husimi function of the field for five different values of $\omega t=\{5,10,15,20,25\}$ is depicted for (b)-(f) isolated dynamics and $(\mathrm{g})-(\mathrm{k})$ open dynamics. The blue and green dots indicate the bottom of the effective potential for the isolated and open cases, respectively. In the simulation we have set $\sqrt{\Omega N / \omega}=100, \kappa=0.1 \omega, \gamma=0.01 \Omega, g=1.03 g_{c}$, and $N=1$.

means that the double-well potential becomes in fact identical to the inverted harmonic oscillator (see Appendix B).

The comparison between the dynamics of the Dicke model and the inverted oscillator is presented in Fig. 3, where we show the number of photons as a function of time [Fig. 3(a)] and squeezing of photons as a function of time for isolated [Figs. 3(b)-3(f)] and open [Figs. 3(b)-3(k)] dynamics through the Husimi function. For the case of isolated dynamics Figs. 3(b) and 3(c) depict squeezing (exponential growth of the photon number) due to the inverted harmonic oscillator potential and its termination as the state starts to feel the potential minima (blue dots). The slowdown of squeezing and reaching of the potential minima can be seen in Figs. 3(d) and 3(e). Finally, Fig. 3(f) depicts antisqueezing (decrease of the number of photons). For the case of open dynamics (see Appendix D), we initially observe similar behavior in Figs. 3(g)-3(i), but with a slower growth of the photon number. Furthermore, as the system dissipates the energy, the state cannot climb back to the local maximum located at $x=0$ and eventually becomes an incoherent mixture of two coherent states with opposite amplitudes (symmetry breaking), which can be seen in Figs. 3(j) and 3(k).

\section{CONCLUSION AND OUTLOOK}

We have shown that the dynamics following a quench from the ground state in the normal phase $(g=0)$ into the superradiant phase in the Dicke model can be described by a model of an inverted harmonic oscillator and argued that in the thermodynamic limit the Hamiltonian of the system becomes equivalent to the inverted harmonic oscillator. The test of results can be readily performed in quantum simulators which can realize the Dicke model [13,32-38] and in quantum simulators which realize the quantum $\operatorname{Rabi}$ model $(N=1)[39,40]$ using various platforms including cold atoms [41], trapped ions [42], superconducting circuits [43], and electrons trapped in liquid helium [44]. The presented mechanism should also apply to an arbitrary quantum system exhibiting a quantum phase transition associated with an effective double-well or sombrero potential.

The presented idea may be used as a tool to study quantum phase transitions [1,45], the quantum Kibble-Zurek mechanism [46-50] both theoretically and experimentally, and the quantum Lieb-Robinson bound for how fast correlations can spread in a quantum system [11]. The results of this work can also be applied in quantum metrology for precise measurements of $\omega$ and $\Omega$ [51-53] as well as for the preparation of squeezed states, simulations of the inflation of the early universe [54-57], the Unruh effect [58,59], Hawking radiation [60], quantum chaos [61,62], and potentially to study many other aspects of physics in which the inverted harmonic oscillator has been harnessed as an underlying mechanism (see Ref. [20] for a recent review). 
Finally, we would like to point out that even though this model bears a resemblance to the Landau theory of phase transition [63], the presented description is fully quantum.

\section{ACKNOWLEDGMENTS}

Simulations were performed using the open-source QuantumOptics.jl framework in JULIA [64]. K.G. acknowledges discussions with Friederike Metz, Ayaka Usui, Lewis Ruks, Farokh Mivehvar, and Michał Baczyk. This work was supported by the Okinawa Institute of Science and Technology Graduate University. K.G. acknowledges support from the Japanese Society for the Promotion of Science (Grant No. P19792).

\section{APPENDIX A: INVARIANCE OF THE INITIAL STATE UNDER TRANSFORMATION}

In order to clearly see the effect of the inverted oscillator, the initial state has to remain unaltered under the transformation

$$
\hat{U}=\exp \left(i \frac{g}{\sqrt{N} \Omega}\left(\hat{a}+\hat{a}^{\dagger}\right) \hat{S}_{y}\right) .
$$

The condition for this to happen can be found by considering the overlap

$$
\left\langle\downarrow 0\left|\exp \left(i \frac{g}{\sqrt{N} \Omega}\left(\hat{a}+\hat{a}^{\dagger}\right) \hat{S}_{y}\right)\right| 0 \downarrow\right\rangle,
$$

where $|0 \downarrow\rangle \equiv|0\rangle \otimes|\downarrow\rangle$ is the ground state of the system for $g=0$. Expanding the exponential function to fourth order (the higher-order terms can be safely neglected because of $1 / N$ dependence), we get

$$
\begin{aligned}
& \left\langle\downarrow 0\left|\exp \left(i \frac{g}{\sqrt{N} \Omega}\left(\hat{a}+\hat{a}^{\dagger}\right) \hat{S}_{y}\right)\right| 0 \downarrow\right\rangle \\
& =\langle\downarrow 0 \mid 0 \downarrow\rangle+\left\langle\downarrow 0\left|i \frac{g}{\sqrt{N} \Omega}\left(\hat{a}+\hat{a}^{\dagger}\right) \hat{S}_{y}\right| 0 \downarrow\right\rangle \\
& \quad+\left\langle\downarrow 0\left|i^{2} \frac{g^{2}}{N \Omega^{2}}\left(\hat{a}+\hat{a}^{\dagger}\right)^{2} \hat{S}_{y}^{2}\right| 0 \downarrow\right\rangle \\
& \quad+\left\langle\downarrow 0\left|i^{3} \frac{g^{3}}{\sqrt{N^{3}} \Omega^{3}}\left(\hat{a}+\hat{a}^{\dagger}\right)^{3} \hat{S}_{y}^{3}\right| 0 \downarrow\right\rangle \\
& \quad+\left\langle\downarrow 0\left|i^{4} \frac{g^{4}}{N^{2} \Omega^{4}}\left(\hat{a}+\hat{a}^{\dagger}\right)^{4} \hat{S}_{y}^{4}\right| 0 \downarrow\right\rangle+O\left(\frac{g^{5}}{\sqrt{N^{5}} \Omega^{5}}\right) .
\end{aligned}
$$

(a)

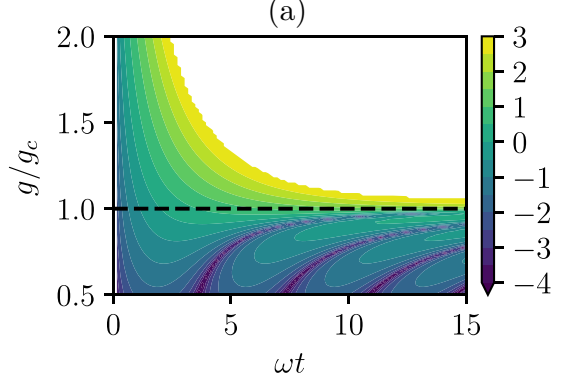

(d)

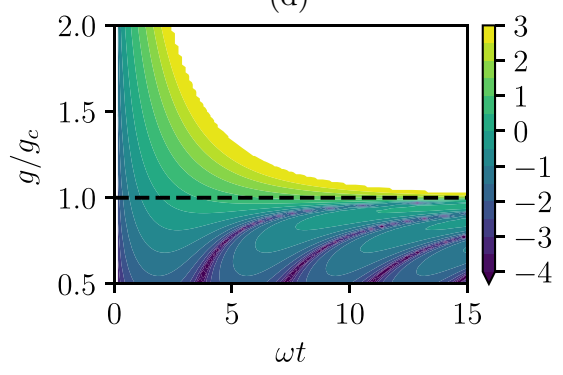

(g)

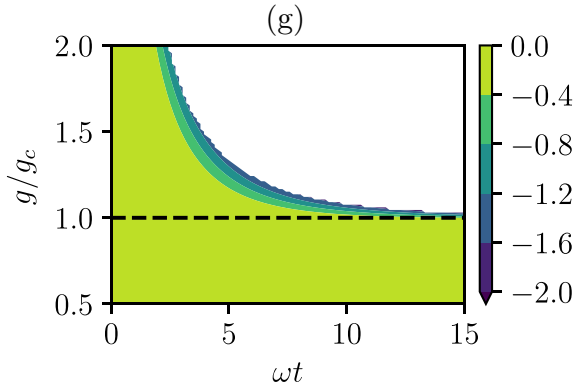

(b)

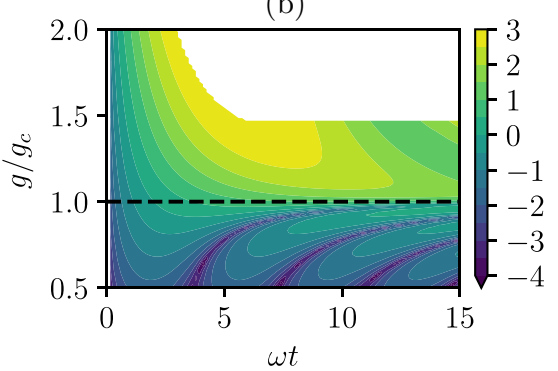

(e)

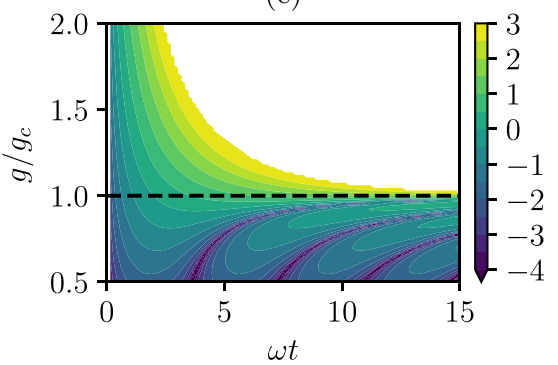

(h)

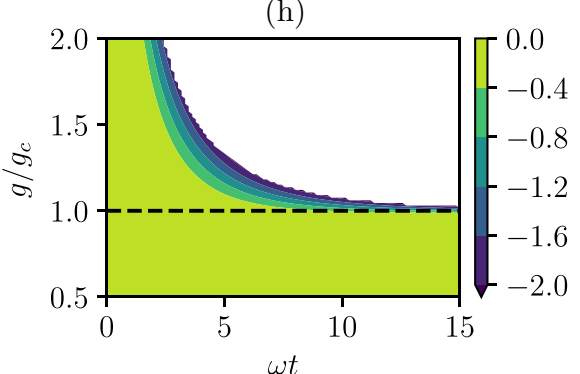

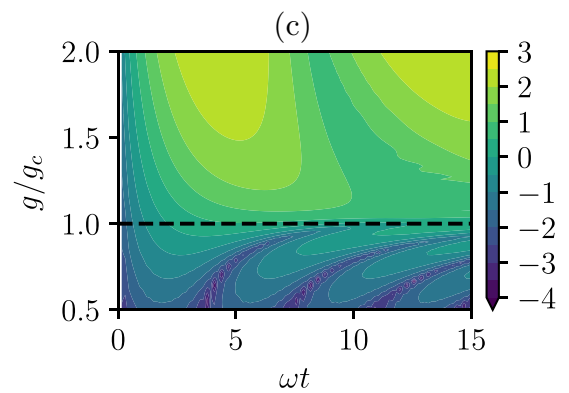

(f)

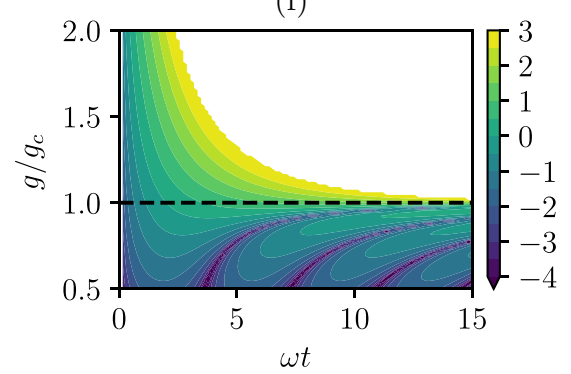

(i)

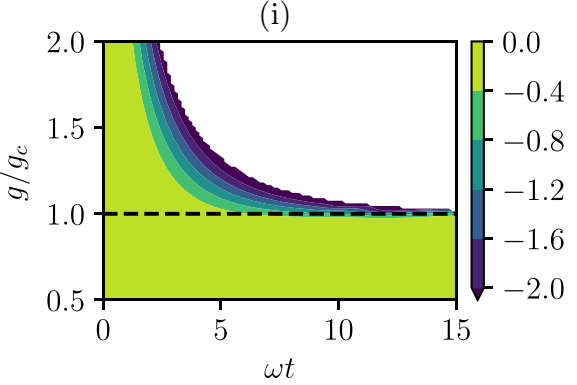

FIG. 4. Extension of Fig. 1. (a)-(c) The Dicke model can be used to simulate the physics of (d)-(f) the inverted harmonic oscillator. (g)-(i) The logarithm of fidelity between the states generated by these two Hamiltonians sets a time limitation for the simulator to work properly. White regions correspond to unreliable numerical simulations after the boundary of the Hilbert space (set to $n=3000$ ) has been reached. 
(a)

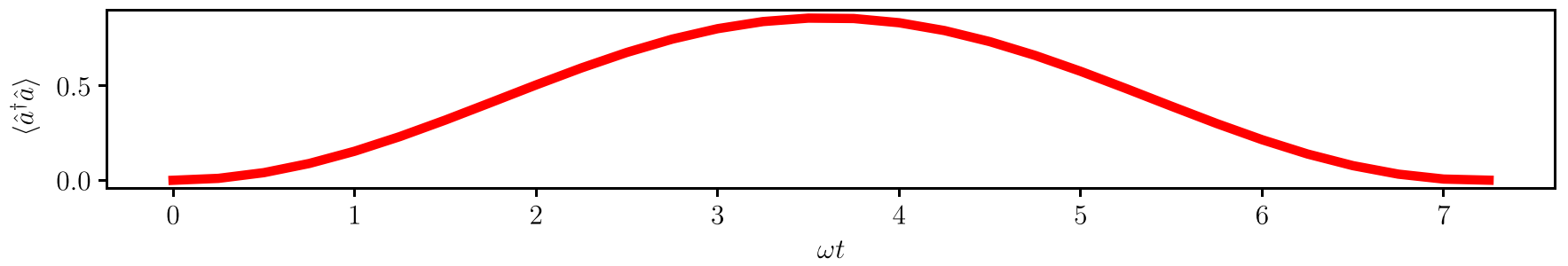

(b)

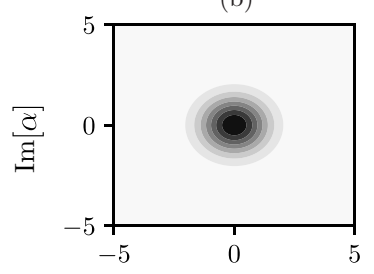

(g)

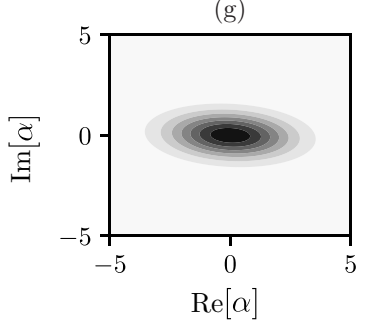

(c)

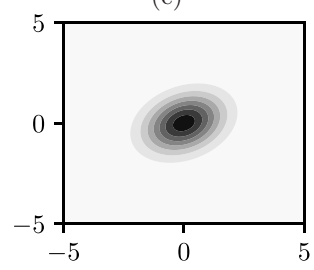

(h)

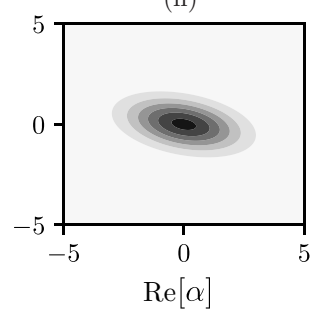

(d)

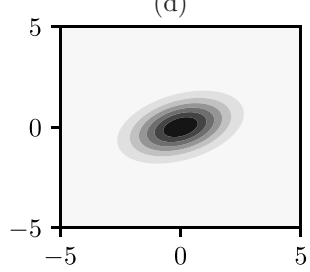

(i)

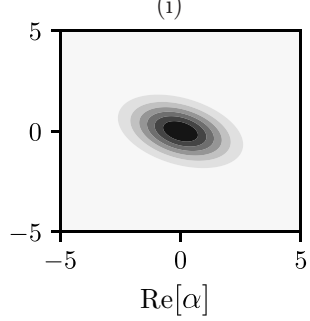

(e)
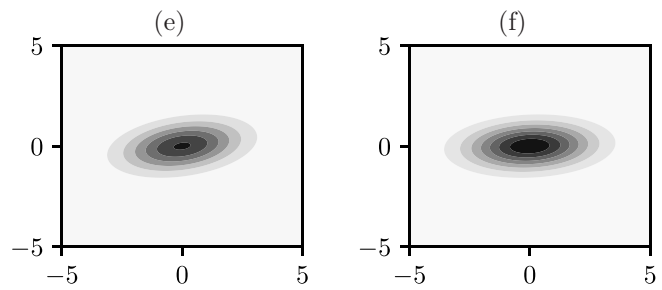

(j)

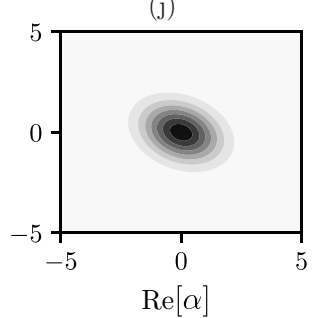

(k)

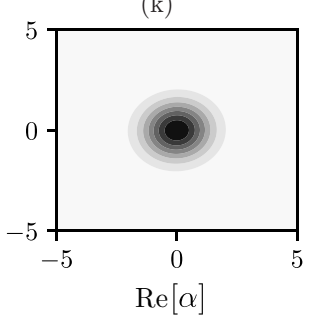

FIG. 5. Squeezing (photon number) oscillations after the quench to the normal phase (cf. Fig. 6). (a) Number of photons as a function of time. (b)-(k) Husimi function of the field for ten different values of $\omega t=\{0,0.8,1.6,2.4,3.2,4.0,4.8,5.6,6.4,7.2\}$, respectively, for isolated dynamics, showing the squeezing oscillations. In the simulation we have set $\sqrt{\Omega N / \omega}=100, g=0.9 g_{c}$, and $N=1$.

Since terms with odd powers yield zero after the evaluation, we can simplify the above overlap to

$$
\begin{aligned}
& \left\langle\downarrow 0\left|\exp \left(i \frac{g}{\sqrt{N} \Omega}\left(\hat{a}+\hat{a}^{\dagger}\right) \hat{S}_{y}\right)\right| 0 \downarrow\right\rangle \\
& \approx 1-\frac{g^{2}}{4 \Omega^{2}}+\frac{g^{4}}{g_{c}^{4}} \frac{\omega^{2}}{\Omega^{2}}\left(\frac{9}{16}-\frac{24}{N}\right) .
\end{aligned}
$$

Therefore, if we want the state $|0 \downarrow\rangle$ to remain invariant, the following condition has to be fulfilled:

$$
\frac{1}{4} \frac{g^{2}}{g_{c}^{2}} \frac{\omega}{\Omega} \ll 1 .
$$

(a)

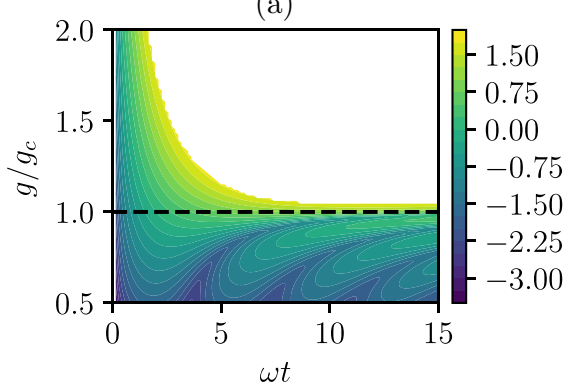

If, however, the initial state is not invariant under the transformation, the system will still simulate the inverted harmonic oscillator but for a transformed state, which though seems impractical. This can be seen explicitly if we transform the Schrödinger equation

$$
i \partial_{t} \hat{U}|\psi\rangle=\hat{H}_{\mathrm{eff}} \hat{U}|\psi\rangle \rightarrow i \partial_{t}|\tilde{\psi}\rangle=\hat{H}_{\mathrm{eff}}|\tilde{\psi}\rangle
$$

FIG. 6. Logarithm of the average number of photons as a function of $g / g_{c}$ and $t$ for (a) $\kappa=0.01 \omega$ and $\gamma=0.1 \Omega$, (b) $\kappa=0.1 \omega$ and $\gamma=0.1 \Omega$, and (c) $\kappa=0.5 \omega$ and $\gamma=0.1 \Omega$. The black dashed line corresponds to the critical coupling in the thermodynamic limit. White regions correspond to unreliable numerical simulations after the boundary of the Hilbert space (set to $n=200$ ) has been reached. In the numerical simulations we set $\sqrt{\Omega N / \omega} \approx 31.6$ and $N=1$. 


\section{APPENDIX B: CONNECTOR OPERATOR}

The condition for simulating the inverted oscillator with the Dicke model can be expressed with the use of the fidelity

$$
\left\langle\psi\left|e^{i \hat{\mathrm{HM}}_{\mathrm{DM}} t} e^{-i \hat{H}_{\mathrm{IO}} t}\right| \psi\right\rangle=e^{i \xi(t)},
$$

where the subscripts DM and IO correspond to the Dicke model and the inverted oscillator, respectively. As discussed in the main text, if the imaginary part of $\xi(t)$ is negligible, one system can simulate the physics of the other one even if the Hamiltonians are different. In Fig. 4 we plot the logarithm of $\left|e^{i \xi(t)}\right|$. When this logarithm is equal to 0 , it means the initial state is an eigenstate of the connector operator

$$
\hat{h}(t)=t\left(\hat{H}_{\mathrm{DM}}-\hat{H}_{\mathrm{IO}}\right)+\frac{i t^{2}}{2}\left[\hat{H}_{\mathrm{DM}},-\hat{H}_{\mathrm{IO}}\right]+\cdots,
$$

with eigenvalue equal to multiples of $\pi$ including 0 . As can be seen in Fig. 4, by increasing $\sqrt{N \Omega / \omega}$, we are increasing the critical time for which the Dicke model can simulate the inverted harmonic oscillator. In the limit of $\sqrt{N \Omega / \omega} \rightarrow \infty$, the Dicke model can realize exactly the same dynamics as the inverted harmonic oscillator. In other words, by increasing $\sqrt{N \Omega / \omega}$ the initial state can remain an eigenstate of the connector operator for longer times as the connector operator becomes an identity in the thermodynamic limit.

\section{APPENDIX C: SQUEEZING OSCILLATIONS IN THE NORMAL PHASE}

In the main text we stated that the Dicke model after the quench to the normal phase exhibits squeezing oscillations or oscillations of the number of photons if the initial state is the field vacuum state. In order to understand it, one has to realize that squeezing a vacuum leads to a state with a nonzero number of photons. Therefore, squeezing and antisqueezing (squeezing oscillations) will lead to oscillations of the number of photons. This can be seen in Fig. 5, where we show the number of photons as a function of time [Fig. 5(a)] and the Husimi function for ten different times depicting a period of squeezing oscillations [Figs. 5(b)-5(k)].

\section{APPENDIX D: EFFECT OF DECOHERENCE}

The discussion from the main text revolves around isolated systems. However, from an experimental perspective, one has to include decoherence which is a consequence of an inability to perfectly isolate a quantum system from its environment. In order to account for these typically unwanted effects, we use the Lindblad master equation approach

$$
\begin{aligned}
\frac{d \hat{\rho}}{d t}= & -i[\hat{H}, \hat{\rho}]+2 \kappa\left(\hat{a} \hat{\rho} \hat{a}^{\dagger}-\left\{\hat{a}^{\dagger} \hat{a}, \hat{\rho}\right\}\right) \\
& +2 \gamma\left(\hat{S}_{-} \hat{\rho} \hat{S}_{+}-\left\{\hat{S}_{+} \hat{S}_{-}, \hat{\rho}\right\}\right),
\end{aligned}
$$

where $\kappa$ and $\gamma$ account for the damping of mode $\hat{a}$ and damping of the spin, respectively, with $\hat{S}_{-}=\hat{S}_{+}^{\dagger}=\hat{S}_{x}-i \hat{S}_{y}$ the spin lowering operator. The effect of spin damping should be negligible as the initial state is the lowest-energy spin eigenstate and for a limited time the system conserves the projection of the spin onto the $z$ axis. If this were not the case, the damping mechanism would transform the state of the spin into its lowest-energy eigenstate and increase the squeezing rate (change the frequency of the inverted oscillator). In both phases the photon damping will cause the system to eventually reach a steady state which is not a vacuum state as the system is driven by a nonzero $g$. The results of the numerical simulations for both phases are presented in Fig. 6, where we plot the average number of photons as a function of $g / g_{c}$ and $t$ for a fixed ratio $\sqrt{\Omega N / \omega}$ for an initial vacuum state evolved with master equation from Eq. (D1) using the Dicke Hamiltonian (1) with three different values of $\kappa$. As expected, by introducing and increasing the photon loss rate $\kappa$, the number of produced photons is reduced and eventually reaches a steady state [see Fig. 6(c)]. Squeezing of photons as a function of time including the inverted harmonic oscillator phase and reaching the potential minima phase (associated with a steady state) is depicted in Figs. 3(g)-3(k).
[1] S. Sachdev, Quantum phase transitions, in Handbook of Magnetism and Advanced Magnetic Materials (Wiley Online Library, New York, 2007).

[2] M. Henkel, H. Hinrichsen, S. Lübeck, and M. Pleimling, NonEquilibrium Phase Transitions (Springer, Dordrecht, 2008), Vol. 1.

[3] J. Dziarmaga, Dynamics of a Quantum Phase Transition: Exact Solution of the Quantum Ising Model, Phys. Rev. Lett. 95, 245701 (2005).

[4] A. Polkovnikov, Universal adiabatic dynamics in the vicinity of a quantum critical point, Phys. Rev. B 72, 161201(R) (2005).

[5] A. Polkovnikov, K. Sengupta, A. Silva, and M. Vengalattore, Colloquium: Nonequilibrium dynamics of closed interacting quantum systems, Rev. Mod. Phys. 83, 863 (2011).

[6] A. Keesling, A. Omran, H. Levine, H. Bernien, H. Pichler, S. Choi, R. Samajdar, S. Schwartz, P. Silvi, S. Sachdev, P. Zoller, M. Endres, M. Greiner, V. Vuletić, and M. D. Lukin, Quantum Kibble-Zurek mechanism and critical dynamics on a programmable Rydberg simulator, Nature (London) 568, 207 (2019).

[7] P. Calabrese and J. Cardy, Time Dependence of Correlation Functions Following a Quantum Quench, Phys. Rev. Lett. 96, 136801 (2006).

[8] L. E. Sadler, J. M. Higbie, S. R. Leslie, M. Vengalattore, and D. M. Stamper-Kurn, Spontaneous symmetry breaking in a quenched ferromagnetic spinor Bose-Einstein condensate, Nature (London) 443, 312 (2006).

[9] C. Kollath, A. M. Läuchli, and E. Altman, Quench Dynamics and Nonequilibrium Phase Diagram of the Bose-Hubbard Model, Phys. Rev. Lett. 98, 180601 (2007).

[10] D. Chen, M. White, C. Borries, and B. DeMarco, Quantum Quench of an Atomic Mott Insulator, Phys. Rev. Lett. 106, 235304 (2011).

[11] M. Cheneau, P. Barmettler, D. Poletti, M. Endres, P. Schauß, T. Fukuhara, C. Gross, I. Bloch, C. Kollath, and S. Kuhr, Light- 
cone-like spreading of correlations in a quantum many-body system, Nature (London) 481, 484 (2012).

[12] M. Heyl, A. Polkovnikov, and S. Kehrein, Dynamical Quantum Phase Transitions in the Transverse-Field Ising Model, Phys. Rev. Lett. 110, 135704 (2013).

[13] J. Klinder, H. Keßler, M. Wolke, L. Mathey, and A. Hemmerich, Dynamical phase transition in the open Dicke model, Proc. Natl. Acad. Sci. USA 112, 3290 (2015).

[14] B. M. Garraway, The Dicke model in quantum optics: Dicke model revisited, Philos. Trans. R. Soc. A 369, 1137 (2011).

[15] C. Emary and T. Brandes, Chaos and the quantum phase transition in the Dicke model, Phys. Rev. E 67, 066203 (2003).

[16] P. Pérez-Fernández, A. Relaño, J. M. Arias, P. Cejnar, J. Dukelsky, and J. E. García-Ramos, Excited-state phase transition and onset of chaos in quantum optical models, Phys. Rev. E 83, 046208 (2011).

[17] J. Chávez-Carlos, M. A. Bastarrachea-Magnani, S. Lerma-Hernández, and J. G. Hirsch, Classical chaos in atom-field systems, Phys. Rev. E 94, 022209 (2016).

[18] R. J. Lewis-Swan, A. Safavi-Naini, J. J. Bollinger, and A. M. Rey, Unifying scrambling, thermalization and entanglement through measurement of fidelity out-of-time-order correlators in the Dicke model, Nat. Commun. 10, 1581 (2019).

[19] A. Jannussis and E. Skuras, Harmonic oscillator with complex frequency, Nuovo Cimento B 94, 29 (1986).

[20] V. Subramanyan, S. S. Hegde, S. Vishveshwara, and B. Bradlyn, Physics of the inverted harmonic oscillator: From the lowest Landau level to event horizons, Ann. Physics, 168470 (2021), DOI: 10.1016/j.aop.2021.168470.

[21] L. Bakemeier, A. Alvermann, and H. Fehske, Quantum phase transition in the Dicke model with critical and noncritical entanglement, Phys. Rev. A 85, 043821 (2012).

[22] M.-J. Hwang, R. Puebla, and M. B. Plenio, Quantum Phase Transition and Universal Dynamics in the Rabi Model, Phys. Rev. Lett. 115, 180404 (2015).

[23] S. Ibáñez, X. Chen, E. Torrontegui, J. G. Muga, and A. Ruschhaupt, Multiple Schrödinger Pictures and Dynamics in Shortcuts to Adiabaticity, Phys. Rev. Lett. 109, 100403 (2012).

[24] K. Gietka, A. Usui, J. Deng, and T. Busch, Simulating the Same Physics with Two Distinct Hamiltonians, Phys. Rev. Lett. 126, 160402 (2021).

[25] R. H. Dicke, Coherence in spontaneous radiation processes, Phys. Rev. 93, 99 (1954).

[26] K. Hepp and E. H. Lieb, On the superradiant phase transition for molecules in a quantized radiation field: The Dicke maser model, Ann. Phys. (NY) 76, 360 (1973).

[27] V. M. Bastidas, C. Emary, B. Regler, and T. Brandes, Nonequilibrium Quantum Phase Transitions in the Dicke Model, Phys. Rev. Lett. 108, 043003 (2012).

[28] R. J. Lewis-Swan, S. R. Muleady, D. Barberena, J. J. Bollinger, and A. M. Rey, Characterizing the dynamical phase diagram of the Dicke model via classical and quantum probes, Phys. Rev. Res. 3, L022020 (2021).

[29] Y. Ashida, A. İmamoğlu, and E. Demler, Cavity Quantum Electrodynamics at Arbitrary Light-Matter Coupling Strengths, Phys. Rev. Lett. 126, 153603 (2021).

[30] T. Holstein and H. Primakoff, Field dependence of the intrinsic domain magnetization of a ferromagnet, Phys. Rev. 58, 1098 (1940).
[31] Z. Zhang and H. Fan, Properties of states generated by excitations on a squeezed vacuum state, Phys. Lett. A 165, 14 (1992).

[32] P. Domokos and H. Ritsch, Collective Cooling and SelfOrganization of Atoms in a Cavity, Phys. Rev. Lett. 89, 253003 (2002).

[33] A. T. Black, H. W. Chan, and V. Vuletić, Observation of Collective Friction Forces due to Spatial Self-Organization of Atoms: From Rayleigh to Bragg Scattering, Phys. Rev. Lett. 91, 203001 (2003).

[34] K. Baumann, C. Guerlin, F. Brennecke, and T. Esslinger, Dicke quantum phase transition with a superfluid gas in an optical cavity, Nature (London) 464, 1301 (2010).

[35] R. Mottl, F. Brennecke, K. Baumann, R. Landig, T. Donner, and $\mathrm{T}$. Esslinger, Roton-type mode softening in a quantum gas with cavity-mediated long-range interactions, Science 336, 1570 (2012).

[36] Z. Zhang, C. H. Lee, R. Kumar, K. J. Arnold, S. J. Masson, A. L. Grimsmo, A. S. Parkins, and M. D. Barrett, Dicke-model simulation via cavity-assisted Raman transitions, Phys. Rev. A 97, 043858 (2018).

[37] I. Aedo and L. Lamata, Analog quantum simulation of generalized Dicke models in trapped ions, Phys. Rev. A 97, 042317 (2018).

[38] C. Hamner, C. Qu, Y. Zhang, J. Chang, M. Gong, C. Zhang, and P. Engels, Dicke-type phase transition in a spin-orbit-coupled Bose-Einstein condensate, Nat. Commun. 5, 4023 (2014).

[39] P. Forn-Díaz, L. Lamata, E. Rico, J. Kono, and E. Solano, Ultrastrong coupling regimes of light-matter interaction, Rev. Mod. Phys. 91, 025005 (2019).

[40] A. F. Kockum, A. Miranowicz, S. De Liberato, S. Savasta, and F. Nori, Ultrastrong coupling between light and matter, Nat. Rev. Phys. 1, 19 (2019).

[41] A. Dareau, Y. Meng, P. Schneeweiss, and A. Rauschenbeutel, Observation of Ultrastrong Spin-Motion Coupling for Cold Atoms in Optical Microtraps, Phys. Rev. Lett. 121, 253603 (2018).

[42] D. Lv, S. An, Z. Liu, J.-N. Zhang, J. S. Pedernales, L. Lamata, E. Solano, and K. Kim, Quantum Simulation of the Quantum Rabi Model in a Trapped Ion, Phys. Rev. X 8, 021027 (2018).

[43] J. Braumüller, M. Marthaler, A. Schneider, A. Stehli, H. Rotzinger, M. Weides, and A. V. Ustinov, Analog quantum simulation of the Rabi model in the ultra-strong coupling regime, Nat. Commun. 8, 779 (2017).

[44] K. M. Yunusova, D. Konstantinov, H. Bouchiat, and A. D. Chepelianskii, Coupling between Rydberg States and Landau Levels of Electrons Trapped on Liquid Helium, Phys. Rev. Lett. 122, 176802 (2019).

[45] W. H. Zurek, U. Dorner, and P. Zoller, Dynamics of a Quantum Phase Transition, Phys. Rev. Lett. 95, 105701 (2005).

[46] T. W. B. Kibble, Topology of cosmic domains and strings, J. Phys. A: Math. Gen. 9, 1387 (1976).

[47] T. W. B. Kibble, Some implications of a cosmological phase transition, Phys. Rep. 67, 183 (1980).

[48] W. H. Zurek, Cosmological experiments in superfluid helium? Nature (London) 317, 505 (1985).

[49] W. H. Zurek, Cosmological experiments in condensed matter systems, Phys. Rep. 276, 177 (1996).

[50] A. Chandran, A. Erez, S. S. Gubser, and S. L. Sondhi, KibbleZurek problem: Universality and the scaling limit, Phys. Rev. B 86, 064304 (2012). 
[51] L. Garbe, M. Bina, A. Keller, M. G. A. Paris, and S. Felicetti, Critical Quantum Metrology with a Finite-Component Quantum Phase Transition, Phys. Rev. Lett. 124, 120504 (2020).

[52] Y. Chu, S. Zhang, B. Yu, and J. Cai, Dynamic Framework for Criticality-Enhanced Quantum Sensing, Phys. Rev. Lett. 126, 010502 (2021).

[53] Authors K. Gietka, L. Ruks, and Th. Busch (unpublished).

[54] A. Albrecht, P. Ferreira, M. Joyce, and T. Prokopec, Inflation and squeezed quantum states, Phys. Rev. D 50, 4807 (1994).

[55] L. P. Grishchuk and Y. V. Sidorov, Squeezed quantum states of relic gravitons and primordial density fluctuations, Phys. Rev. D 42, 3413 (1990).

[56] A. H. Guth and S.-Y. Pi, Quantum mechanics of the scalar field in the new inflationary universe, Phys. Rev. D 32, 1899 (1985).

[57] S. Eckel, A. Kumar, T. Jacobson, I. B. Spielman, and G. K. Campbell, A Rapidly Expanding Bose-Einstein Condensate: An Expanding Universe in the Lab, Phys. Rev. X 8, 021021 (2018).
[58] W. G. Unruh, Notes on black-hole evaporation, Phys. Rev. D 14, 870 (1976).

[59] L. C. B. Crispino, A. Higuchi, and G. E. A. Matsas, The Unruh effect and its applications, Rev. Mod. Phys. 80, 787 (2008).

[60] S. W. Hawking, Black hole explosions? Nature (London) 248, 30 (1974).

[61] K. Hashimoto, K.-B. Huh, K.-Y. Kim, and R. Watanabe, Exponential growth of out-of-time-order correlator without chaos: Inverted harmonic oscillator, J. High Energy Phys 11 (2020) 068.

[62] A. Bhattacharyya, W. Chemissany, S. S. Haque, J. Murugan, and B. Yan, The multi-faceted inverted harmonic oscillator: Chaos and complexity, SciPost Phys. Core 4, 2 (2021).

[63] L. D. Landau, On the theory of phase transitions, Zh. Eksp. Teor. Fiz. 11, 19 (1937).

[64] S. Krämer, D. Plankensteiner, L. Ostermann, and H. Ritsch, QuantumOptics.jl: A Julia framework for simulating open quantum systems, Comput. Phys. Commun. 227, 109 (2018). 